\title{
DESCRIPTION DE TROIS NOUVELLES ESPECES de Paractenopsyllus (Siphonaptera : Leptopsyllinae) de Madagascar
}

\author{
DUCHEMIN J.B.* \& RATOVONJATO J.*
}

Summary: DESCRIPTION OF THRFF NFW SPECIFS

of Paractenopsylles genus (Siphonaptera: Leptopsyllinae) FROM MADAGASCAR

The males of three new and rare species of the Malagasy endemic genus Paractenopsyllus Wagner, 1938 are described. Paractenopsyllus beaucournui, $P$. oconnori and $P$. raxworthyi have been collected on endemic small mammals and the black rat. As for others species of the genus, biotopes are rain-forests of the central highlands or the northern mountains of Madagascar. Two of the described species were collected at elevations lower than typical for the genus and below the limits of the highland bioclimatic zone. Morphological affinities between these new species and those already described provide taxonomic framework for the genus. An updated identification key is provided for the diagnosis of all the 20 Malagasy species of Leptopsyllinae.

KEY WORDS : Paractenopsyllus, new species, Madagascar

\section{Résumé :}

Les mâles de trois nouvelles espèces rares de Paractenopsyllus Wagner, 1938, genre endémique de Madagascar, sont décrits. Paractenopsyllus beaucournui, $P$. oconnori et $P$. raxworthyi ont été collectés à partir de micromammifères endémiques de Madagascar (excepté un rat noir) et, comme les autres espèces du genre, les biotopes de récolte se situent au sein des forêts des hautes terres centrales ou des massifs du Nord de lîle. Deux des trois espèces décrites ont été récoltées à des altitudes relativement basses pour le genre, à la limite de la zone écoclimatique des hautes terres. Les affinités morphologiques entre ces nouvelles espèces et celles déjà décrites permettent des rapprochements taxonomiques. Une clef de détermination des 20 espèces de Leptopsyllinae malgaches est présentée.

MOTS CLÉS : Paractenopsyllus, espèces nouvelles, Madagascar

\section{INTRODUCTION}

P lusieurs prospections récentes d'inventaire faunistique dans les forêts malgaches (Goodman, 1996, 1998) ont permis la description de plusieurs nouvelles espèces de puces du genre Paractenopsyllus Wagner, 1938 appartenant à la sous-famille des Leptopsyllinae (Duchemin, 2003, 2004). La radiation spécifique de ce genre endémique est forte sur Madagascar, île-continent. Nous présentons ici la description des mâles de trois nouvelles espèces qui augmente le nombre d'espèces du genre à 17 . Les places respectives des trois espèces, ici décrites au sein du genre, sont discutées ainsi que les limites de la technique de prospection utilisée.

\footnotetext{
* Unité d'entomologie médicale, Institut Pasteur de Madagascar, BP 1274, Antananarivo 101, Madagascar.

Correspondance: J.B. Duchemin, Cermes, BP 10887, Niamey, Niger. Tel. : 00227752045 - Fax : 00227753180

E-mail: duchemin@cermes.ne
}

\section{RÉSULTATS}

\section{PARACTENOPSYLLUS BEAUCOURNUI N. SP}

- Matériel examiné

Un seul spécimen mâle holotype récolté sur un rat noir Rattus rattus (Linnaeus, 1758) ( ${ }^{\circ}$ BMOC 95 203-083), dans le massif d'Anjahanaribe-Sud $\left(49^{\circ} 26,5^{\prime} \mathrm{E}, 12^{\circ}\right.$ $\left.44,5^{\prime} \mathrm{S}\right)$, province d'Antsiranana, Madagascar, à une altitude de $1550 \mathrm{~m}$, en novembre 1994, par Barry O'Connor.

- Étymologie

Cette espèce est nommée d'après le Professeur JeanClaude Beaucournu, de l'Université de Rennes, France, qui, patiemment, a guidé nos pas sur le chemin peu fréquenté de la taxonomie des puces.

- Dépôt du type

Holotype déposé dans les collections du Field Museum of Natural History, Chicago, USA.

- Diagnose

Cette espèce est remarquable parmi les espèces du genre Paractenopsyllus par ses petits peignes dorsaux 

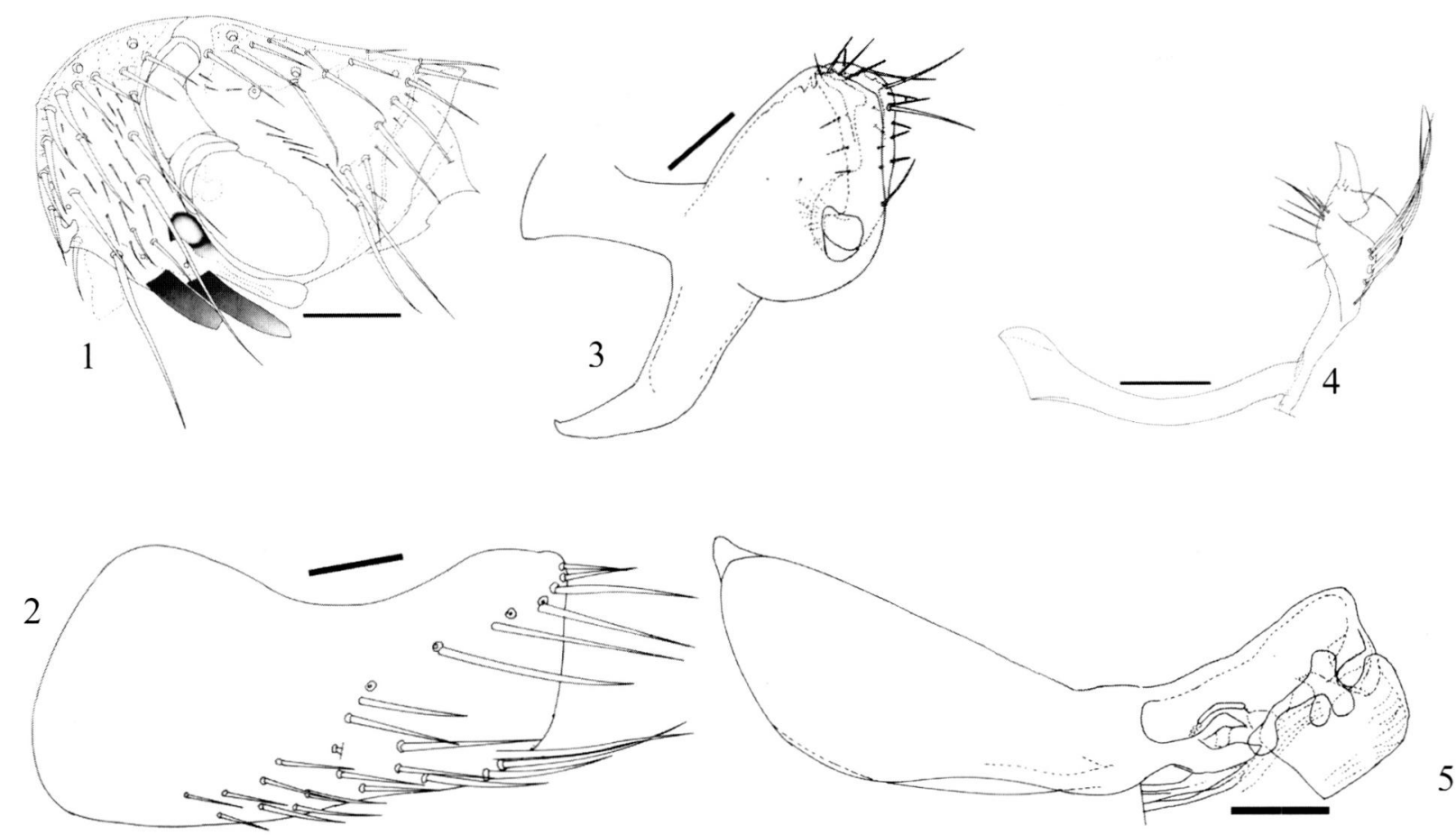

Figs 1-5. - Paractenopsyllus beaucournui $\mathrm{n}$. sp.

1 : tête du mâle; 2 : sternite VIII 3 : clasper; 4 : sternite IX; 5 : phallosome.

Échelle $=0,1 \mathrm{~mm}$.

sur les quatre premiers tergites abdominaux. Seul P. viettei Klein, 1965 porte parfois plus d'une spinule marginale par côté et par segment, mais se différencie de $P$. beaucournui par ses caractères sexuels (sternite IX et phallosome).

\section{- Description}

Tête (Fig. 1). Tubercule frontal situé un peu en dessous du milieu du front. La rangée frontale est composée de six soies dont les deux plus basses sont épaissies mais non spiniformes et de trois soies sub-marginales. Six soies longues au-dessus des pièces buccales chez le mâle. Le peigne génal est composé de deux épines. Une soie est insérée derrière le clypeus au-dessus de l'insertion des palpes maxillaires. L'œil est sinueux dans sa partie interne et inférieure. Quatre rangs de soies occipitales incluant un rang sub-marginal de sept soies, un antérieur de quatre, un moyen de deux et un postérieur de cinq soies, avec une soie unique dans l'angle postéroinférieur de l'occiput. Les palpes labiaux atteignent le milieu de la hauteur de la coxa I.

Thorax. Prothorax avec deux rangées de soies, l'antérieure composée de 12 soies pour les deux côtés, tout comme la rangée postérieure qui porte des soies plus longues. Le peigne pronotal se compose de 26 épines. Mésothorax avec quatre rangs de soies et trois pseudosoies, deux dorsales et une ventrale. Métathorax avec cinq rangées de soies. Quatre spinules marginales sur le bord postérieur (deux de chaque côté). Métépiméron avec trois rangées de soies, une antérieure de quatre, une moyenne de quatre et une soie postérieure. Faux peigne tibial présent et composé de 13 soies fortes et trois soies plus longues.

Abdomen (segments non modifiés). Premier tergite avec trois rangs, le principal et postérieur composé de dix soies, 4-5 spinules marginales de chaque côté, formant une petit peigne dorso-latéral. Sur les autres segments : trois rangs sur les $2^{\text {ème }}$ et $3^{\text {ème }}$ tergites et deux rangs du $4^{\text {ème }}$ au $7^{\text {ème }}$, portant 13 ou 14 soies. Trois à quatre spinules marginales sur chaque côté des $2^{\text {ème }}$,

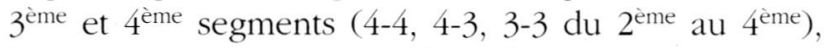
formant d'autres peignes dorso-latéraux. Trois soies antépygidiales avec les rapports de longueurs suivants (en partant de la plus dorsale, et la médiane étant prise comme dénominateur): 0.36/1/0.36. Sternites: sternite II avec une soie de chaque côté; sternite III-VI chacun avec une seule rangée de $2-3$ soies avec $2-4$ soies plus courtes en avant.

Segments modifiés. Tergite VIII avec trois longues soies, un bord supérieur avec une convexité bien marquée et un angle apical inférieur à $90^{\circ}$.

Sternite VIII (Fig. 2) avec un bord supérieur concave. Le bord apical est droit dans sa moitié supérieure puis 
dessine un angle très arrondi dans sa moitié inférieure. L'angle supérieur est à $90^{\circ}$ et porte deux soies sub-marginales courtes et fortes. Deux à trois soies relativement longues dans l'angle inférieur, à distance du bord. Sur la partie latérale du sternite VIII, cinq soies longues sont disposées selon une ligne oblique vers le bas et l'avant de la moitié supérieure, tandis que quatre soies plus courtes prolongent cette rangée vers le bas. Environ une douzaine de soies courtes le long du bord inférieur.

Segment IX (Fig. 3). Manubrium court; apex du basimère large et anguleux, dessinant une bosse sur le bord postérieur. Soie acétabulaire relativement courte.

Télomère court, s'élargissant nettement vers son extrémité dont la largeur est approximativement double de celle de la base. L'angle antérieur de l'apex du télomère est arrondi et projeté vers l'avant. Deux longues soies sur le bord postérieur du télomère, au niveau de la bosse sur le bord postérieur du basimère.

Sternite IX (Fig. 4). Branche proximale globalement concave vers le haut, avec la dilation proximale projetée vers l'avant, son bord supérieur se continuant avec le bord supérieur de la branche proximale principale, sans angle bien marqué. Branche distale avec une forme triangulaire à base supérieure. Seulement cinq soies sur le bord postérieur dont les trois supé- rieures sont particulièrement longues, dépassant l'extrémité du lobe distal.

Phallosome (Fig. 5). Plaque pénienne particulièrement large. L'apex du lobe dorsal est également large avec un lobe ventral peu marqué. Hamulus massif et large aussi bien sur son bras proximal que distal; le bras proximal présente, sur son bord antérieur, des lobules entourant partiellement le bras distal du sternite IX. Longueur (insecte monté sur lame) : $2,85 \mathrm{~mm}$.

\section{PARACTENOPSYLLUS OCONNORI N. SP.}

\section{- Matériel examiné}

Un spécimen mâle holotype, récolté sur Eliurus tanala Major, 1896 ( $\mathrm{N}^{\circ}$ : BMOC 940325 032), provenant du Parc National d'Andringitra ( $47^{\circ} 00^{\prime}$ E, $22^{\circ} 14^{\prime} \mathrm{S}$ ), province de Fianarantsoa, Madagascar à une altitude de $1000 \mathrm{~m}$, en novembre 1993, par Barry O'Connor. Un spécimen mâle paratype, récolté sur Eliurus webbi Ellerman, 1949 ( $\mathrm{N}^{\circ}$ : BMOC 940325035$)$, provenant du même site, même date, même récolteur.

\section{- Étymologie}

Cette espèce est nommée d'après Barry O'Connor, acarologiste du Museum of Zoology, University of Michigan, Ann Arbor, USA, qui m'a très aimablement fait parvenir ses spécimens de puces récoltées à partir de mammifères de forêts naturelles malgaches.
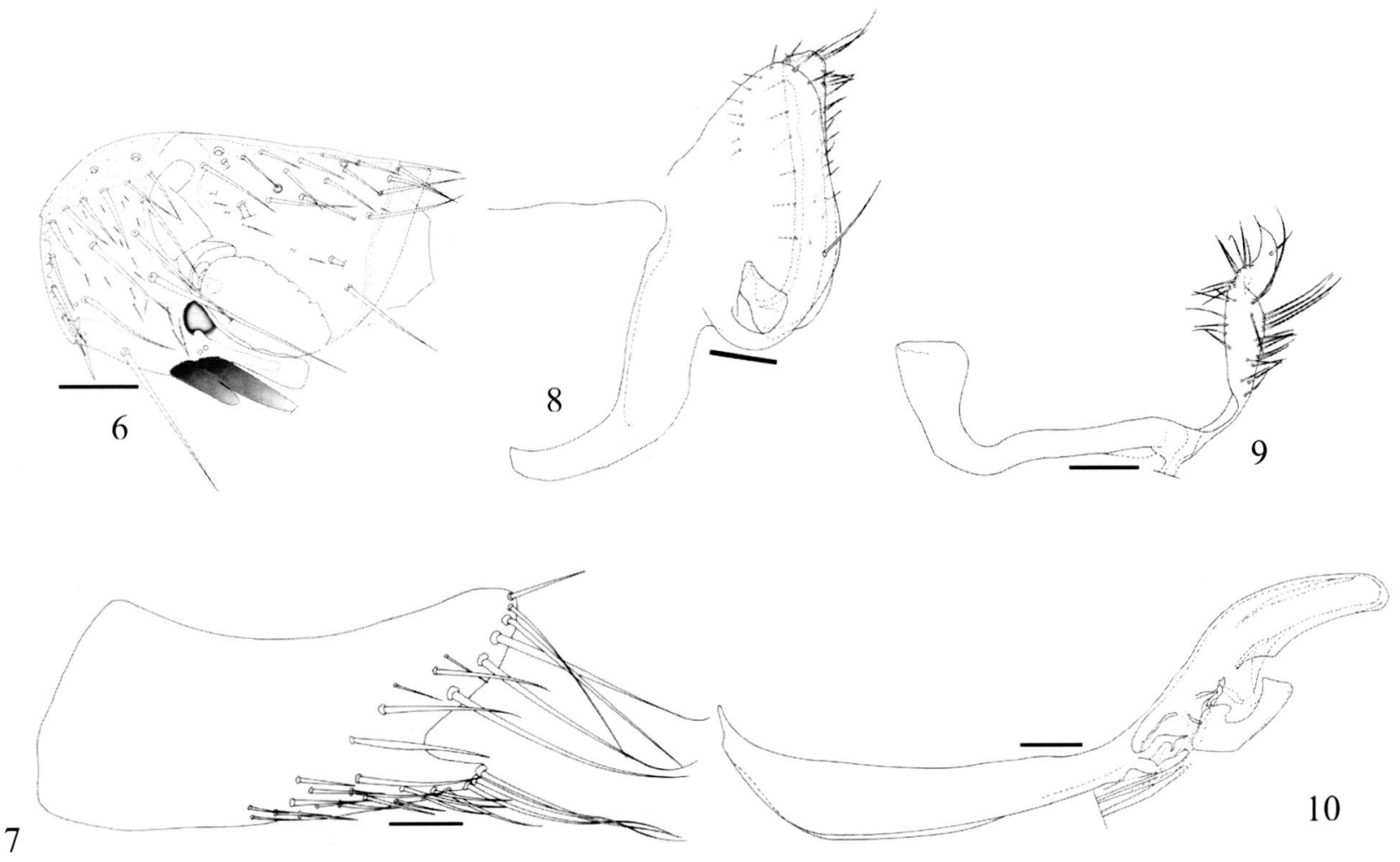

Figs 6-10. - Paractenopsyllus oconnori n. sp.

6: tête du mâle; 7 : sternite VIII; 8 : clasper; 9 : sternite IX; 10 : phallosome.

Échelle $=0,1 \mathrm{~mm}$. 
- Dépôt des types

Holotype et paratype déposés dans la collection du Field Museum of Natural History, Chicago, USA.

- Diagnose

Cette espèce est originale au sein des espèces du genre et est très facilement reconnaissable grâce au lobe dorsal du phallosome très allongé. Cette forme de phallosome la rapproche des espèces telles que P. rouxi Duchemin, 2004, P. vauceli Klein, 1965 et P. grandidieri Klein, 1965.

- Description

Tête (Fig. 6). Le tubercule frontal est situé un peu en dessous du milieu du front. La rangée frontale est composée de 5-6 soies dont les deux premières sont à peine plus fortes que les autres mais non spiniformes et de trois soies sub-marginales. Six soies longues au dessus des pièces buccales chez le male. Le peigne génal est composé de deux épines. Une soie est insérée derrière le clypeus au dessus de l'insertion des palpes maxillaires. L'œil est sinueux dans sa partie interne et inférieure. Quatre rangs de soies occipitales incluant un rang sub-marginal de six soies avec un net hiatus entre la plus basse et celle immédiatement au dessus, un antérieur de 4-5, un moyen de 2-3 et un postérieur de 6-7 soies, avec une soie unique dans l'angle postéro-inférieur de l'occiput. Les palpes labiaux atteignent le milieu de la hauteur de la coxa I.

Thorax. Prothorax avec deux rangées de soies, l'antérieure composé de 14-15 soies pour les deux côtés, la rangée postérieure et principale portant 12 soies plus longues. Le peigne pronotal est composé de 3233 épines. Mésothorax avec $4-5$ rangs de soies et trois pseudo-soies, deux dorsales et une ventrale, cette dernière étant doublée d'un côté sur le paratype. Métathorax avec quatre rangées de soies. Deux spinules marginales sur le bord postérieur (une de chaque côté). Métépiméron avec trois rangées de soies, une antérieure de 4-5, une moyenne de 4-5 et une ou deux soies postérieures. Faux peigne tibial présent et composé de 15-16 soies fortes et trois soies plus longues. Abdomen (segments non modifiés). Premier tergite avec trois rangées, la principale et postérieure composée de 9-10 soies avec une spinule marginale de chaque côté du bord postérieur, dans sa partie supérieure. Sur les autres segments : 2-3 rangs de 1315 soies, constamment deux rangs du $5^{\text {ème }}$ au $7^{\text {ème }}$ tergite, constamment trois rangs sur le $2^{\text {ème }}$ tergite. Une spinule marginale de chaque côté du $2^{\text {ème }}$ au $4^{\text {ème }}$ segments. Trois soies antépygidiales avec les ratios suivants (en partant de la plus dorsale) : 0.35/1/0.4. Sternites : sternite II avec une soie de chaque côté; sternite III-VI chacun avec une rangée principale de 3-4 soies et 2-3 soies plus courtes en avant.

Segments modifiés : Tergite VIII avec trois longues soies, un bord supérieur légèrement convexe, un angle apical supérieur à $90^{\circ}$ et un bord postérieur et inférieur très convexe.

Sternite VIII (Fig. 7) avec un bord supérieur légèrement concave. Le bord apical présente une nette concavité dans sa moitié inférieure avec cinq soies longues dans sa moitié supérieure, surmontées d'une soie plus fine dans l'angle supérieur et 2-3 soies longues dans l'angle inférieur, surmontées de $0-2$ soies plus fines. Latéralement 5-6 soies moyennes ou courtes alignées très obliquement vers l'avant et vers le bas avec, le long du bord inférieur du sternite VIII, une quinzaine de soies courtes.

Segment IX (Fig. 8). Apophyse antéro-dorsale implantée haut sur le basimère, donnant une apparence allongée au manubrium. Apex du basimère arrondi; soie acétabulaire relativement longue pour le genre. Télomère long et droit, se rétrécissant de la base vers l'apex carré. Les soies les plus longues du bord postérieur sont situées dans l'angle postérieur de l'apex du télomère.

Sternite IX (Fig. 9). Branche proximale sinueuse avec une jonction très arrondie avec la dilatation proximale d'aspect trapézoïdal. Branche distale de forme allongée avec les bords antérieurs et postérieurs convexes; la sétation abondante sur le bord postérieur présente des soies fines et plus longues près de l'angle postérosupérieur. Le lobe distal est allongé à l'apex et globuleux à la base.

Phallosome (Fig. 10). Le lobe apico-dorsal a une forme générale en $\mathrm{S}$ allongé avec un apex régulièrement arrondi lui conférant un aspect tubulaire caractéristique. Le lobe ventral est très réduit, en triangle. Hamulus plutôt allongé, l'apex du bras distal est modérément dilaté et de forme carrée.

Longueurs (insectes montés sur lame): mâle $3,7 \mathrm{~mm}$ (holotype), 3,3 $\mathrm{mm}$ (paratype).

PARACTENOPSYLLUS RAXWORTHYI N. SP.

- Matériel examiné

Un spécimen mâle holotype, récolté sur Tenrec ecaudatus (Schreber, 1777) ( ${ }^{\circ}$ BMOC 950701003$)$, provenant de la Montagne d'Ambre ( $49^{\circ} 10^{\prime} \mathrm{E}, 12^{\circ} 31^{\prime} \mathrm{S}$ ), province d'Antsiranana, Madagascar à une altitude de 1000 m, en mars 1994, par Barry O'Connor.

Un spécimen mâle paratype, récolté sur Microgale brevicaudata G. Grandidier, 1899 ( $\mathrm{N}^{\circ} \mathrm{RAX} 3960$ ), provenant de la forêt de Bezavona ( $49^{\circ} 52^{\prime} \mathrm{E}, 13^{\circ} 32^{\prime} \mathrm{S}$ ), province d'Antsiranana, Madagascar à une altitude de 550 m, en Février 2002, par l'équipe de Christopher J. Raxworthy.

- Étymologie

Cette espèce est nommée d'après Christopher J. Raxworthy, herpétologue de l'American Museum of Natural History, New York, USA. 


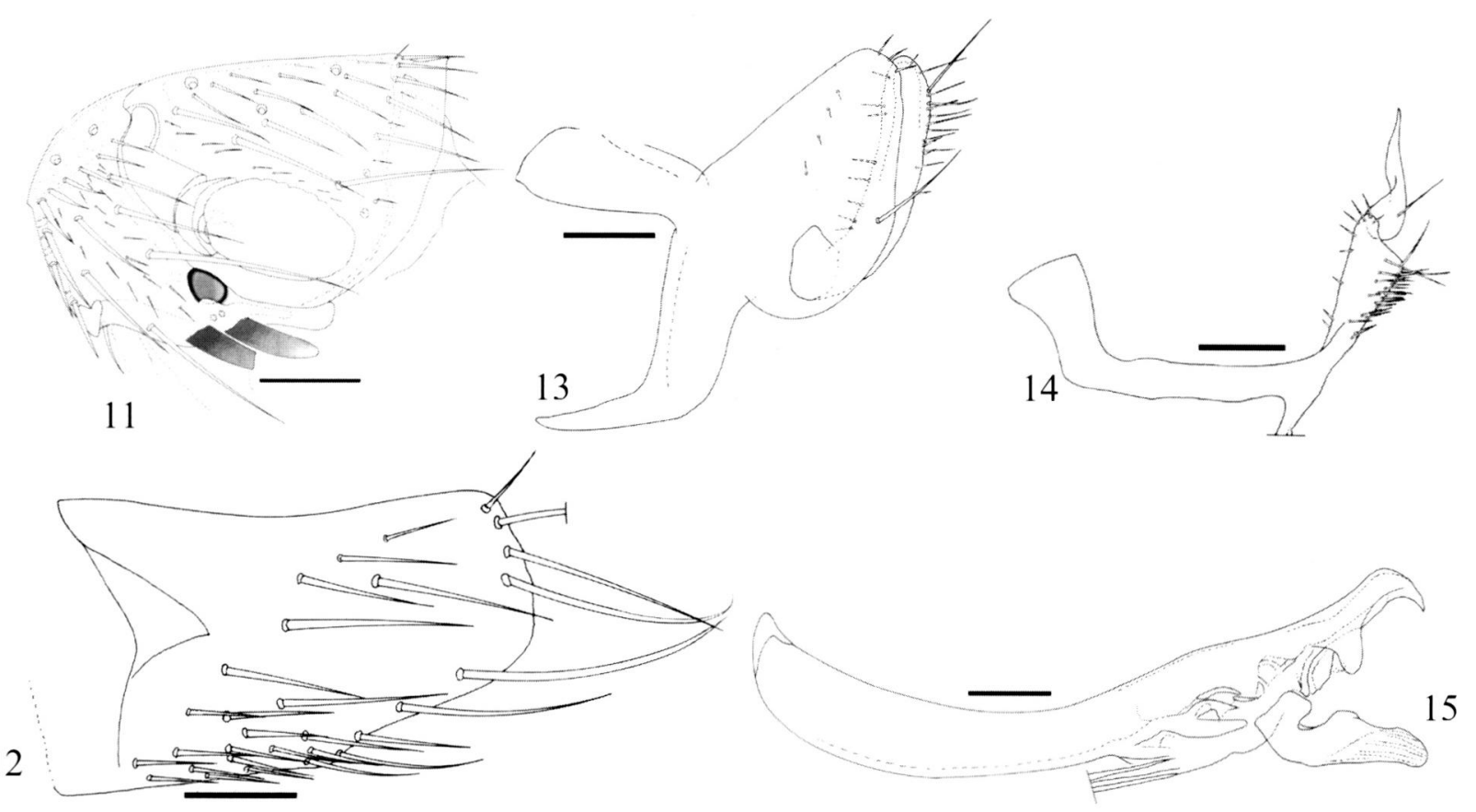

Figs 11-15. - Paractenopsyllus raxworthyi $\mathrm{n}$. sp.

11 : tête du mâle; 12 : sternite VIII; 13 : clasper; 14 : sternite IX; 15 : phallosome.

Échelle $=0,1 \mathrm{~mm}$

- Dépôt des types

Holotype déposé dans la collection du Field Museum of Natural History, Chicago, USA. Paratype conservé provisoirement en alcool à l'Institut Pasteur de Madagascar, Antananarivo, Madagascar.

\section{- Diagnose}

Cette espèce est proche de Paractenopsyllus randrianasoloi Klein, 1968 et $P$. gemelli Duchemin, 2004. Le lobe dorsal, tout comme ces deux dernières espèces est en crochet, mais le peigne pronotal porte un nombre de dents nettement plus faible que $P$. randrianasoloi et $P$. gemelli. L'apex en crochet du lobe dorsal est bien séparé des lobes ventraux, tandis que le bord apical du $8^{\text {ème }}$ sternite est droit et non pas arrondi. Enfin l'altitude de récolte du paratype correspond à la plus basse notée pour une espèce du genre.

\section{- Description}

Tête (Fig. 11). Le tubercule frontal est situé un peu en dessous du milieu du front. La rangée frontale est composée de six soies dont la première est spiniforme et de trois soies sub-marginales dont la plus haute est la plus longue. Six soies longues au-dessus des pièces buccales chez le mâle. Le peigne génal est composé de deux épines. Une soie insérée derrière le clypeus au-dessus de l'insertion des palpes maxillaires. L'œil est sinueux dans sa partie interne et inférieure. Quatre rangs de soies occipitales incluant un rang sub-marginal de sept soies, un antérieur de 4-5, un moyen de trois et un postérieur de six soies, avec une soie unique dans l'angle postéro-inférieur de l'occiput. Les palpes labiaux atteignent le milieu de la hauteur de la coxa I.

Thorax. Prothorax avec deux rangées de soies, l'antérieure composé de 12 soies pour les deux côtés, ainsi que la rangée postérieure mais portant des soies plus longues. Le peigne pronotal porte 24 épines. Mésothorax avec cinq rangs de soies et trois pseudo-soies, deux dorsales et une ventrale. Métathorax avec quatre rangées de soies. Deux spinules marginales sur le bord postérieur (une de chaque côté). Métépiméron avec trois rangées de soies, une antérieure de quatre, une moyenne de quatre et deux soies postérieures. Faux peigne tibial présent et composé de 13 soies fortes et trois soies plus longues.

Abdomen (segments non modifiés). Premier tergite avec trois rangées, la principale et postérieure composée de neuf soies avec une spinule marginale de chaque côté du bord postérieur, dans sa partie supérieure. Sur les autres segments : trois rangs du $2^{\text {ème }}$ au $5^{\text {ème }}$ tergite, et deux rangs sur les 6 ème et 7 ème segments, portant $12-13$ soies. Une spinule marginale de chaque côté du $2^{\text {ème }}$ au $4^{\text {ème }}$ segments. Trois soies antépygidiales avec les ratios suivants (comme précédemment) : 0.25/1/0.3. Sternites : sternite II avec une soie de chaque côté; sternite III-VI chacun avec une seule rangée de trois soies avec 1-3 soies plus courtes en avant. 
Segments modifiés. Tergite VIII avec trois longues soies, un bord supérieur convexe et un angle apical arrondi, inférieur à $90^{\circ}$.

Sternite VIII (Fig. 12) avec un bord supérieur droit. Le bord apical également droit porte quatre soies dans sa moitié supérieure, les deux plus basses sont plus longues. Pas de soies à l'angle inférieur arrondi. Le sternite VIII porte latéralement huit à dix soies moyennes et une quinzaine de soies le long du bord inférieur, courtes pour la plupart, mais les postérieures nettement plus longues. Segment IX (Fig. 13) Manubrium court; apex du basimère relativement étroit et arrondi; soie acétabulaire longue. Télomère peu incurvé, à bords parallèles et apex arrondi. Une seule soie longue remarquable sur le bord postérieur, à peine plus courte que la soie acétabulaire.

Sternite IX (Fig. 14). Branche proximale avec une dilatation proximale trapézoïdale à bord antérieur concave. Branche distale de forme triangulaire à base supérieure. Environ une vingtaine de soies courtes, celles près de l'angle supérieur plus longues. Le lobe distal est très allongé, en forme de point d'interrogation renversé, avec un apex étroit.

Phallosome (Fig. 15). Extrémité du lobe dorsal en forme de crochet, avec un lobe ventral arrondi. La distance entre l'extrémité du crochet et le lobe ventral est plus longue que la largeur de ce lobe. Hamulus avec un bras distal long et large, strié sur sa partie postérieure, un apex arrondi et un lobe dorsal marqué. Longueur (insecte monté sur lame) : $2,7 \mathrm{~mm}$.

\section{CLEF D'IDENTIFICATION DES ESPÈCES CONNUES DE LEPTOPSYLLINAE DE MADAGASCAR}

L a clef des espèces de Paractenopsyllus la plus récente était celle de Hopkins \& Rothschild (1971) et ne traitait que six espèces, alors que celle de Klein \& Grenier (1967), bien que plus ancienne, permettait la diagnose de huit espèces. Afin d'inclure les dernières espèces décrites, une nouvelle clef morphologique a été construite pour la diagnose des 20 espèces de Leptopsyllinae de Madagascar, incluant Leptopsylla segnis (Schönherr, 1811), espèce cosmopolite et les deux genres endémiques Paractenopsyllus et Tsaractenus Klein, 1968.

1 - Dents génales $=4 \ldots \ldots \ldots \ldots \ldots \ldots \ldots$.Leptopsylla segnis

- Dents génales $=2$, exceptionnellement 3 3.........2

2 - 3 soies sur le bord inférieur du fémur postérieur

- 2 soies sur le bord inférieur du fémur postérieur
3 - Apophyse génale très large, à peu près égale à la largeur des deux dents génales réunies

.Tsaratenus rodhaini

- Apophyse génale étroite, nettement moins large que les deux dents du peigne génal .............T. grenieri

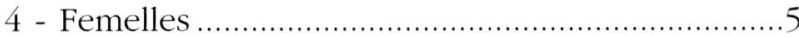

- Mâles ................................................................... 18

5 - 2 lobes sur le bord postérieur du sternite VII ...6

- 1 seul lobe sur le bord postérieur du sternite VII

6 - Peigne pronotal $>28$ dents (pour les deux côtés) ....Paractenopsyllus (Paractenopsyllus) kerguistel

- Peigne pronotal $\leq 28$ dents (pour les deux côtés)

- Spermathèque nettement allongée (long $=2$ $\times \operatorname{larg})$............................................. (P.) vauceli

- Spermathèque arrondie (long $<1,5$ long) ..........8

8 - 5 soies longues au dessus des pièces buccales..9

- 4 soies longues au dessus des pièces buccales..10

9 - 2 lobes postérieurs du sternite VII arrondis et réguliers.................................... (P.) duplantieri

- 2 lobes postérieur du sternite VII crochus P. (P.) juliamarinus

10 - Au moins 3 soies frontales spiniformes ...........11

- Au plus 2 soies frontales nettement spiniformes. 12

11 - Ductus bursae dessinant un hémicadre plus ou moins rectangulaire ouvert vers l'arrière, lobe inférieur du sternite VII allongé ........P. (P.) petiti

- Ductus bursae avec 4 courbes, lobe inférieur du sternite VII court et triangulaire.......P. (P.) ratovonjatoi

12 - Encoche supérieure du sternite VII nettement plus étroite que le lobe sus-jacent ......P. (P.) pauliani

- Encoche supérieure du sternite VII nettement plus large que le lobe sus-jacent .......P. (P.) albignaci

13 - Toutes les soies de la série frontale fines et non modifiées......................................................... 14

- Au moins une soie frontale épaissie ................16

14 - Perula très dilatée

P. (Consobrinopsyllus) goodmani

- Perula de diamètre proche de celui des ducti (2 à $3 \times)$

15 - Lobe du sternite VII à extrémité arrondie et dépassant le bord inférieur des sternites.......P. (P.) rouxi

- Lobe du sternite VII à extrémité anguleuse P. (P.) grandidieri

16 - Lobe du sternite VII à extrémité fine et anguleuse ..................................................................... viei

- Lobe du sternite VII à extrémité arrondie ........17

17 - Bulga plus large à l'insertion de la hilla qu'au dessus de l'area cribriformis...P. (P.) randrianasoloi

- Bulga aussi large à ces deux niveaux..P. (P.) gemelli

- Mâles

18 - Bord postérieur du sternite VIII avec une concavité ou un lobe 
- Bord postérieur du sternite VIII droit ou arrondi, sans concavité .........................................24

19 - Epines du peigne pronotal < 29 ..................20

- Epines du peigne pronotal > $30 \ldots \ldots \ldots \ldots \ldots \ldots \ldots . . \ldots 21$

20 - Hamulus large, échancré d'une cavité régulièrement arrondie ................................ (P.) viettet

- Hamulus différent......................... (P.) albignaci

21 - Apex du lobe dorsal du phallosome arrondi...22 - Apex du lobe dorsal du phallosome en crochet P. (P.) randrianasoloi (pro parte)

22 - Lobe ventral du phallosome peu ou pas développé..... P. (P.) oconnori

- Lobe ventral du phallosome développé ...........23

23 - Apex du basimère large ..........P. (P.) grandidieri - Apex du basimère étroit.................... (P.) rouxi

24 - Bord postérieur du sternite VIII nettement plus étroit que la largeur du sternite

.25

- Bord à peu près aussi large que la largeur du sternite 26

25 - Manubrium du basimère nettement plus long que le corps du basimère ................ (P.) duplantieri

- Manubrium du basimère aussi long que le corps du basimère $P$. (P.) juliamarinus

26 - Encoche sclérotisée à la jonction du bras distal et proximal du sternite IX ............P. (C.) goodmani

- Pas d'encoche à cette jonction ........................27

27 - Epines du peigne pronotal $>28 \ldots \ldots \ldots \ldots \ldots \ldots \ldots .28$

- Epines du peigne pronotal $\leq 28 \ldots \ldots \ldots \ldots \ldots \ldots \ldots . . . . .30$

28 - Apex du lobe dorsal du phallosome en crochet.

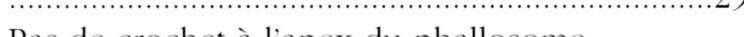

Pas de crochet à l'apex du phallosome. P. (P.) kerguisteli

29 - Crochet court, inférieur à la moitié de la largeur de l'apex du phallosome................ (P.) gemelli

- Crochet fin et long, supérieur ou égal à la largeur de l'apex du phallosome.

P. (P.) randrianasoloi (pro parte)

30 - Premiers segments abdominaux avec 2 à 4 épines dorsales....

..31

- Premiers segments abdominaux avec plus de 6 épines dorsales. .P. (P.) beaucournui

31 - Apex du lobe dorsal du phallosome en crochet. P. (P.) raxworthyi

- Apex du lobe dorsal différent. 32

32 - Au moins 4 soies frontales modifiées spiniformes

- Moins de 4 soies frontales spiniformes............34

33 - Apex du lobe dorsal du phallosome en doigt de

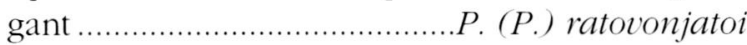
- Apex du lobe dorsal du phallosome différent..... ............................................... (P.) petiti

34 - Apex du lobe dorsal du phallosome large et arrondi ........................................... pauliani

- Apex du lobe dorsal du phallosome arrondi avec un lobe ventral prononcé ............... (P.) vauceli

\section{DISCUSSION}

L a radiation spécifique du genre Paractenopsyllus à Madagascar est manifeste. Le très faible nombre de spécimens présentés ici ne permet pas un complément d'analyse approfondi des hypothèses éventuelles de spéciation. Le seul élément qui pourrait sembler important est l'altitude de récolte relativement basse $(550-1000 \mathrm{~m})$ pour deux des trois espèces décrites, $P$. raxworthyi et $P$. oconnori. Concernant $P$. oconnori, le site de sa récolte, le massif de l'Andringitra, avait permis de retrouver $P$. rouxi à une altitude supérieure. Ces deux dernières espèces présentent une morphologie du phallosome proche, notamment le lobe apico-dorsal, avec une taille, un nombre d'épines du peigne pronotal comparables et aucune soie spiniforme sur la rangée frontale. $P$. raxworthyi est à rapprocher de manière évidente de $P$. randrianasolo $i$ et de $P$. gemelli. Ces trois espèces ont en commun, outre le lobe apico-dorsal du phallosome en crochet, le fait d'avoir été capturées, exclusivement ou souvent, sur des rongeurs du genre Eliurus, dans la région Nord de l'île, même si P. randrianasoloi étend sa répartition sur la marge orientale des hautes terres centrales. Il est important de noter que, parmi ces trois espèces, $P$. raxworthyi possède un nombre d'épines du peigne pronotal nettement plus faible que les deux autres taxons. Il pourrait être justifié de rassembler ces trois espèces très proches au sein d'un groupe, cependant nous pensons prématuré de les rassembler, en l'état actuel des connaissances, au sein d'un sous-genre. La troisième espèce décrite ici, P. beaucournui, a également été capturée dans le nord de l'île. Ses affinités morphologiques sont plus délicates à établir. La description de la femelle permettrait d'établir de tels liens, tandis que la détermination de son hôte primitif, obligatoirement autre que $R$ rattus introduit, pourra faire discuter la présence des pseudo-peignes dorsaux et éclairer le débat sur le rôle de ces organes de protection (Marshall, 1980) ou d'accrochage (Traub, 1980) ou divers (Smit, 1972). Sur le plan de la systématique, les caractères morphologiques des trois nouvelles espèces les font ranger au sein du sous-genre Paractenopsyllus sensu stricto (Duchemin, 2003). Enfin il parâ̂t important de préciser que le mode de récolte des spécimens, par recherche différée d'ectoparasites sur des spécimens conservés en formol dans un linge fin et bien que ne permettant pas une récolte d'un nombre important de spécimens, liée à la fuite précoce classique des puces après la mort de l'animal, a permis de décrire de nouvelles espèces, probablement rares. La conservation en formol, puis en alcool, a permis la conservation des puces permettant leur description, avec une chétotaxie relativement bien conservée. Par contre, ce mode de conser- 
vation interdit toute étude de biologie moléculaire qui pourrait apporter des arguments quant à la position systématique des ces espèces.

\section{REMERCIEMENTS}

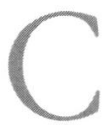

es travaux ont été financés par le World Wildlife Fund (WWF), l'Institut Pasteur de Madagascar (IPM) et la NSF (C. Raxworthy). Nous remercions le Dr Suzanne Chanteau, responsable du programme Peste, le Pr Jean-Felix Roux et le Dr Philippe Mauclère, directeurs de l'IPM pour avoir encouragé les travaux taxinomiques sur les puces. Nous remercions le Pr Jean-Claude Beaucournu (Faculté de Médecine, Rennes, France) pour son éclairage fréquent et patient sur la taxinomie et la systématique des puces et la relecture critique. Steve M. Goodman (Field Museum of Natural History, Chicago, USA and WWFMadagascar) a collecté et identifié les mammifères et permis le contact avec Barry O'Connor (Museum of Zoology, University of Michigan, Ann Arbor, USA) et Christopher Raxworthy. Nous remercions particulièrement Edmond "Dadajao" Rajaonarivelo pour le montage des spécimens.

\section{RÉFÉRENCES}

Beaucolrnu J.-C.\& Fontenille D. Contribution à un catalogue des puces de Madagascar. Archives de l'Institut Pasteur de Madagascar, 1993, édition spéciale, 1-48.

Duchemin J.B. Two new fleas (Siphonaptera: Ceratophyllidae: Leptopsyllinae) of Madagascar: Tsaractenus rodbaini n. sp. and Paractenopsyllus (Consobrinopsyllus n. subgen.) goodmani n.sp. Parasite, 2003, 10, 351-358.

Duchemin J.B. Leptopsyllines from Madagascar (Insecta: Siphonaptera: Ceratophyllidae): description of five new species of Paractenopsyllus Wagner, 1938. Parasite, 2004, 11, 59-70.

Goodman S.M. Floral and faunal inventory of the eastern slopes of the Reserve Naturelle Integrale d'Andringitra, Madagascar with reference to elevational variation. Fieldiana, 1996, 85, 1-319.

GoOdman S.M. Floral and faunal inventory of the Reserve Speciale d'Anjanaharibe-Sud, Madagascar with reference to elevational variation. Fieldiana, 1998, 90, 1-246.

Hopkins G.H.E. \& Rothschild M. An illustrated catalogue of the Rothschild collection of fleas (Siphonaptera) in the British Museum (Natural History). Vol. V: Leptopsyllidae and Ancistropsyllidae. British Museum, 1971, 530 pp., 30 pl.

Klein J.M. \& Grenier P. Description complémentaire de Paractenopsyllus kerguisteli Wagner, 1938 et clé illustrée de détermination des espèces du genre Paractenopsyllus Wagner (Siphonaptera). Cahiers de l'ORSTOM, série. Entomolgie Médicale Parasitologie, 1969, 7 (4), 329-339.
Marshall A.G. The function of combs in ectoparasitic insects, in : Fleas, proceedings of the international conference on fleas. Ashton Wold, Peterborough, UK, Traub R \& Starcke H. (eds). A.A. Balkema, Rotterdam, 1980, 79-87.

Smit F.G.A.M. On some adaptative structures in Siphonaptera. Folia Parasitologica (Praha), 1972, 19, 5-17.

Traub R. Some adaptative modification in fleas, in : Fleas, proceedings of the international conference on fleas, Ashton Wold, Peterborough, UK, Traub R \& Starcke H. (eds). A.A. Balkema, Rotterdam, 1980, 79-87.

WAGNer J. Sur un Cténopsyllide nouveau de Madagascar, Paractenopsyllus kerguisteli n. gen., n. sp. Bulletin de la Société de Pathologie Exotique, 1938, 31 (3), 224-228.

Reçu le 29 septembre 2003 Accepté le 15 décembre 2003 